\title{
BMJ Global Health Protecting children in low-income and middle-income countries from COVID-19
}

\begin{abstract}
Salahuddin Ahmed (D) , ${ }^{1,2}$ Tisungane Mvalo, ${ }^{3,4}$ Samuel Akech, ${ }^{5}$ Ambrose Agweyu, ${ }^{5}$ Kevin Baker, ${ }^{6}$ Naor Bar-Zeev, ${ }^{7}$ Harry Campbell, ${ }^{2,8}$ William Checkley, ${ }^{9}$ Mohammod Jobayer Chisti, ${ }^{10}$ Tim Colbourn (D) , ${ }^{11}$ Steve Cunningham, ${ }^{2,12}$ Trevor Duke, ${ }^{13,14,15}$ Mike English (D) , ${ }^{5,16}$ Adegoke G Falade, ${ }^{17}$ Nicholas SS Fancourt (D) , ${ }^{18}$ Amy S Ginsburg, ${ }^{19}$ Hamish R Graham (D) ,20,21 Diane M Gray, ${ }^{22}$ Madhu Gupta, ${ }^{23}$ Laura Hammitt, ${ }^{7}$ Anneke C Hesseling, ${ }^{24}$ Shubhada Hooli (iD) , ${ }^{25}$ Abdul-Wahab BR Johnson, ${ }^{26}$ Carina King, ${ }^{27}$ Miles A Kirby, ${ }^{28,29}$ Claudio F Lanata, ${ }^{30,31}$ Norman Lufesi, ${ }^{32}$ Grant A Mackenzie, ${ }^{33,34,35}$ John P McCracken, ${ }^{36}$ Peter P Moschovis (D) , ${ }^{37}$ Harish Nair, ${ }^{2,8}$ Osawaru Oviawe, ${ }^{38}$ William S Pomat, ${ }^{39}$ Mathuram Santosham, ${ }^{7}$ James A Seddon, ${ }^{24,40}$ Lineo Keneuoe Thahane, ${ }^{41,42,43}$ Brian Wahl (D) ,7 Marieke Van der Zalm, ${ }^{44}$ Charl Verwey, ${ }^{45,46}$ Lay-Myint Yoshida, ${ }^{47}$ Heather J Zar, ${ }^{48,49}$ Stephen RC Howie, ${ }^{50}$ Eric D McCollum (iD 7,51
\end{abstract}

\begin{abstract}
To cite: Ahmed S, Mvalo T, Akech S, et al. Protecting children in low-income and middle-income countries from COVID-19. BMJ Global Health 2020;5:e002844. doi:10.1136/ bmjgh-2020-002844
\end{abstract}

SA and TM contributed equally.

Received 6 May 2020 Accepted 8 May 2020

Check for updates

(C) Author(s) (or their employer(s)) 2020. Re-use permitted under CC BY-NC. No commercial re-use. See rights and permissions. Published by BMJ.

For numbered affiliations see end of article.

Correspondence to Dr Eric D McCollum; emccoll3@jhmi.edu and Dr Stephen RC Howie; stephen.howie@auckland.ac.nz
A saving grace of the COVID-19 pandemic in high-income and upper middle-income countries has been the relative sparing of children. As the disease spreads across low-income and middle-income countries (LMICs), long-standing system vulnerabilities may tragically manifest, and we worry that children will be increasingly impacted, both directly and indirectly. Drawing on our shared child pneumonia experience globally, we highlight these potential impacts on children in LMICs and propose actions for a collective response.

Current data suggest children are susceptible to severe acute respiratory syndrome coronavirus 2 (SARS-CoV-2) infection but are less likely than adults to become severely ill. $^{1-4}$ Although at first glance these data appear reassuring, the child pneumonia, and broader global child health, experience provides a forewarning of what may be coming in LMICs. High-income countries (HICs) have an under-5 pneumonia mortality rate of 3 per $100000 .^{5}$ In contrast, the rate in LMICs is 200 per 100000 population, with pneumonia the leading infectious cause of under-5 death globally. ${ }^{5}$ While yet unknown, COVID-19, a viral pneumonia syndrome, may impact children in LMICs more severely than what has been observed to date.
The risk factors for poor outcomes in pneumonia are overwhelmingly more prevalent in LMICs than HICs. These include severe malnutrition, low immunisation uptake, nutritional anaemia, HIV exposure or infection, air pollution, poverty, low parental education and, crucially, limited access to high-quality acute healthcare ${ }^{67} \mathrm{In}$ HICs, vulnerable children are being actively 'shielded' from infection, but in LMICs, most will remain exposed.

The indirect effects of the COVID-19 response need attention as they are an enormous threat to the well-being of children. These include widespread parental unemployment, disrupted education, food and housing insecurity and threats to vital preventive health programmes, like immunisation, antenatal care, infant feeding and mental health. The acute care workforce may soon be overwhelmed by COVID-19 needs, and we anticipate critical healthcare services being diverted away from mothers and children. Delays in care seeking may worsen, resulting in more severe illness. So, while transmission of respiratory pathogens may be slowed by pandemic response measures, these measures could accentuate well-established risk factors for poor paediatric outcomes and undermine healthcare systems' abilities to respond. 
What can be done to protect children in LMICs? First, we must prevent the collapse of vital acute care (oxygen, antibiotics, personal protective equipment (PPE)), preventive services (immunisation, maternity care, breastfeeding and nutrition programmes, HIV and malaria prevention) and supply chains and take opportunities for system strengthening that could be a legacy of the pandemic. Maintaining a critical workforce in maternal and child health, enabling healthcare workers to use PPE and empowering community health workers to engage with communities in the response are essential. These measures, taken locally with decisive international support, are likely to save more children's lives than advanced intensive care and should benefit children with COVID-19 and other illnesses, including pneumonia.

Second, COVID-19 testing in LMICs needs massive upscaling and outreach. If we are to understand paediatric COVID-19, surveillance systems-both pandemic and pneumonia focused-need investment for rapid scale-up and testing of children with respiratory illnesses for SARS-CoV-2, testing paediatric contacts of adult cases and accurately reporting child deaths.

Third, pandemic lockdown strategies should maintain vital access to care and be tailored to the particular social, economic and health environments of LMICs. This may not mean following the approach of HICs. Rather, LMICs should also build on their experience and expertise gained during other epidemics and make decisions based on their reality, workforce capacity, population density and migration patterns. Examples could be establishing separate areas in clinics for preventative care by dedicated non-respiratory staff, home vaccination visits, outposts responsible for delivery of household essentials to COVID-19 affected households, retraining newly unemployed people to assist with case finding and contact tracing and sewing groups to produce face masks to support widespread mask strategies.

Fourth, research is key to better understanding COVID-19 fundamentals on children, younger and older, including their role in transmission dynamics, spectrum of illness and outcomes, the impact of comorbidities and common coinfections (viral, bacterial, mycobacterial and parasitic) and how broader pandemic responses impact on health behaviours and outcomes. To accomplish this requires COVID-19 surveillance and rapid cycle research on the effects of pandemic response strategies and context-informed modelling using the best available data and locally relevant assumptions. Understanding COVID-19 in children is essential to developing informed, nuanced pandemic responses, including eventual vaccination strategies. These efforts must be country-driven, network-building, joint global initiatives supported by the international community for the benefit of all.

We expect children in LMICs may be seriously impacted by COVID-19, potentially both directly and indirectly. Balanced strategies that protect children must be central to coordinated and cooperative global pandemic response efforts.

\section{Author affiliations}

${ }^{1}$ Projahnmo Research Foundation, Dhaka, Bangladesh

${ }^{2}$ NIHR Global Health Unit on Respiratory Health (RESPIRE), London, United Kingdom

${ }^{3}$ University of North Carolina Project Malawi, Lilongwe, Malawi

${ }^{4}$ Department of Pediatrics, University of North Carolina-Chapel Hill, Chapel Hill,

North Carolina, USA

${ }^{5}$ KEMRI-Wellcome Trust Research Programme, Nairobi, Kenya

${ }^{6}$ Malaria Consortium, London, United Kingdom

${ }^{7}$ Department of International Health, Johns Hopkins Bloomberg School of Public Health, Baltimore, Maryland, USA

${ }^{8}$ Center for Global Health, Usher Institute, University of Edinburgh Medical School, Edinburgh, United Kingdom

${ }^{9}$ Division of Pulmonary and Critical Care, Department of Medicine, Johns Hopkins University School of Medicine, Baltimore, Maryland, USA

${ }^{10}$ Dhaka Hospital, Nutrition and Clinical Services Division, International Centre for Diarrhoeal Disease and Research, Bangladesh (icddr,b), Dhaka, Bangladesh

${ }^{11}$ Global Health Institute, University College London, London, United Kingdom

${ }^{12}$ Centre for Inflammation Research, University of Edinburgh, Edinburgh, United Kingdom

${ }^{13}$ Paediatric Intensive Care Unit, Royal Children's Hospital, Melbourne, Victoria, Australia

${ }^{14}$ Department of Paediatrics, University of Melbourne, Melbourne, Victoria, Australia

${ }^{15}$ School of Medicine and Health Sciences, University of Papua New Guinea,

Goroka, Papua New Guinea

${ }^{16}$ Centre for Tropical Medicine and Global Health, Nuffield Department of Medicine, University of Oxford, Oxfordshire, United Kingdom

${ }^{17}$ Division of Paediatric Pulmonology, Department of Paediatrics, College of Medicine and University College Hospital, Ibadan, Nigeria

${ }^{18}$ Global and Tropical Health Division, Menzies School of Health Research, Charles Darwin University, Darwin, Northern Territory, Australia

${ }^{19}$ Clinical Trial Center, University of Washington, Seattle, United States

${ }^{20}$ Centre for International Child Health, MCRI, University of Melbourne, Melbourne, Victoria, Australia

${ }^{21}$ Department of Paediatrics, University College Hospital Ibadan, Ibadan, Nigeria

${ }^{22}$ Division Paediatric Pulmonology, Department of Paediatrics, University of Cape Town, Cape Town, South Africa

${ }^{23}$ Department of Community Medicine and School of Public Health, Postgraduate Institute of Medical Education and Research, Chandigarh, India

${ }^{24}$ Desmond Tutu TB Centre, Department of Paediatrics and Child Health, Faculty of Medicine and Health Sciences, Stellenbosch University, Cape Town, South Africa

${ }^{25}$ Department of Pediatrics, Section of Pediatric Emergency Medicine, Baylor

College of Medicine, Houston, United States

${ }^{26}$ Pulmonology \& Infectious Disease Unit, Department of Paediatrics \& Child Health, University of Ilorin/University of llorin Teaching Hospital, llorin, Nigeria

${ }^{27}$ Department of Global Public Health, Karolinska Institutet, Stockholm, Sweden

${ }^{28}$ Gangarosa Department of Environmental Health, Rollins School of Public Health,

Emory University, Atlanta, Georgia, United States

${ }^{29}$ Department of Global Health and Population, Harvard T.H. Chan School of Public Health, Boston, United States

${ }^{30}$ Instituto de Investigación Nutricional, Lima, Peru

${ }^{31}$ Department of Pediatrics, School of Medicine, Vanderbilt University, Nashville, Tennessee, United States

${ }^{32}$ Community Health Sciences Unit, Malawi Ministry of Health, Lilongwe, Malawi

${ }^{33}$ MRC Unit, The Gambia at LSHTM, Fajara, Gambia

${ }^{34}$ Faculty of Infectious \& Tropical Diseases, LSHTM, London, United Kingdom

${ }^{35}$ Murdoch Children's Research Institute, Melbourne, Victoria, Australia

${ }^{36}$ Center for Health Studies, Universidad del Valle de Guatemala, Guatemala City, Guatemala

${ }^{37}$ Divisions of Pulmonary Medicine and Global Health, Department of Pediatrics,

Massachusetts General Hospital, Boston, Massachusetts, USA

${ }^{38}$ Department of Child Health, University of Benin Teaching Hospital, Benin City, Nigeria

${ }^{39}$ Papua New Guinea Institute of Medical Research, Goroka, Papua New Guinea

${ }^{40}$ Department of Infectious Diseases, Imperial College London, London, United Kingdom

${ }^{41}$ Baylor College of Medicine Children's Foundation - Lesotho, Maseru, Lesotho 
${ }^{42}$ Department of Pediatrics, Baylor College of Medicine, Houston, Texas, USA

${ }^{43}$ The International Pediatric AIDS Initiative (BIPAl) at Texas Children's Hospital, Baylor College of Medicine, Houston, Texas, USA

${ }^{44}$ Department of Paediatrics and Child Health, Stellenbosch University, Cape Town, South Africa

${ }^{45}$ Division of Paediatric Pulmonology, Department of Paediatrics, Chris Hani Baragwanath Academic Hospital, Faculty of Health Sciences, University of the Witwatersrand, Johannesburg, South Africa

${ }^{46}$ Respiratory and Meningeal Pathogens Research Unit, Medical Research Council, University of the Witwatersrand, Johannesburg, South Africa

${ }^{47}$ Department of Pediatric Infectious Diseases, Institute of Tropical Medicine, Nagasaki University, Nagasaki, Japan

${ }^{48}$ Department of Paediatrics and Child Health, Red Cross War Memorial Children's Hospital, University of Cape Town, Cape Town, South Africa

${ }^{49}$ SA-MRC Unit on Child and Adolescent Health, University of Cape Town, Cape Town, South Africa

${ }^{50}$ Department of Paediatrics: Child \& Youth Health, University of Auckland, Auckland, New Zealand

${ }^{51}$ Johns Hopkins Global Program in Pediatric Respiratory Sciences, Eudowood Division of Pediatric Respiratory Sciences, Department of Pediatrics, Johns Hopkins School of Medicine, Baltimore, Maryland, USA

Twitter Mike English @ProfMikeEnglish, Nicholas SS Fancourt @fantwit, Hamish R Graham @grahamhamish, Shubhada Hooli @ShubhadaH and Eric D McCollum @tinylungsglobal

Contributors EDM, SA and TM wrote the first draft of the comment. EDM, SA, TM, SRCH, NBZ, HRG, CK and ME provided critical review and editing of comment. All authors reviewed subsequent drafts and agreed on the final version of this comment.

Funding The authors have not declared a specific grant for this research from any funding agency in the public, commercial or not-for-profit sectors.

Competing interests None declared.

Patient and public involvement Patients and/or the public were involved in the design, or conduct, or reporting, or dissemination plans of this research. Refer to the Methods section for further details.

Patient consent for publication Not required.

Provenance and peer review Not commissioned; internally peer reviewed.

Open access This is an open access article distributed in accordance with the Creative Commons Attribution Non Commercial (CC BY-NC 4.0) license, which permits others to distribute, remix, adapt, build upon this work non-commercially, and license their derivative works on different terms, provided the original work is properly cited, appropriate credit is given, any changes made indicated, and the use is non-commercial. See: http://creativecommons.org/licenses/by-nc/4.0/.

\section{ORCID iDs}

Salahuddin Ahmed http://orcid.org/0000-0001-6771-0638

Tim Colbourn http://orcid.org/0000-0002-6917-6552

Mike English http://orcid.org/0000-0002-7427-0826

Nicholas SS Fancourt http://orcid.org/0000-0002-1772-9960

Hamish R Graham http://orcid.org/0000-0003-2461-0463

Shubhada Hooli http://orcid.org/0000-0003-4596-448X

Peter P Moschovis http://orcid.org/0000-0002-9664-5959

Brian Wahl http://orcid.org/0000-0002-0037-7364

Eric D McCollum http://orcid.org/0000-0002-1872-5566

\section{REFERENCES}

1 Dong Y, Mo X, Hu Y, et al. Epidemiology of COVID-19 among children in China. Pediatrics 2020:e20200702.

2 Lu X, Zhang L, Du H, et al. SARS-CoV-2 infection in children. N Engl J Med 2020;382:1663-5.

3 CDC COVID-19 Response Team. Coronavirus Disease 2019 in Children - United States, February 12-April 2, 2020. MMWR Morb Mortal Wkly Rep 2020;69:422-6.

4 Tagarro A, Epalza C, Santos M, et al. Screening and severity of coronavirus disease 2019 (COVID-19) in children in Madrid, Spain. JAMA Pediatr 2020. doi:10.1001/jamapediatrics.2020.1346. [Epub ahead of print: 08 Apr 2020].

5 GBD 2017 Lower Respiratory Infections Collaborators. Quantifying risks and interventions that have affected the burden of lower respiratory infections among children younger than 5 years: an analysis for the global burden of disease study 2017. Lancet Infect Dis 2020;20:60-79.

6 Sonego M, Pellegrin MC, Becker G, et al. Risk factors for mortality from acute lower respiratory infections (ALRI) in children under five years of age in low and middle-income countries: a systematic review and meta-analysis of observational studies. PLoS One 2015; 10:e0116380.

7 Lazzerini M, Sonego M, Pellegrin MC. Hypoxaemia as a mortality risk factor in acute lower respiratory infections in children in low and middle-income countries: systematic review and meta-analysis. PLOS One 2015;10:e0136166. 